\title{
Alpha-I-fucosidase: a novel serum biomarker to predict prognosis in early stage esophageal squamous cell carcinoma
}

\author{
Xiangyang Yu ${ }^{1,2 \#}$, Rusi Zhang ${ }^{1,2 \#}$, Tianzhen Yang ${ }^{1,2 \#}$, Mengqi Zhang ${ }^{3}$, Kexing Xi ${ }^{4}$, Yongbin Lin ${ }^{1,2}$, \\ Yingsheng Wen ${ }^{1,2}$, Gongming Wang ${ }^{1,2}$, Zirui Huang ${ }^{1,2}$, Xuewen Zhang ${ }^{1,5}$, Lanjun Zhang ${ }^{1,2}$ \\ ${ }^{1}$ State Key Laboratory of Oncology in South China, Collaborative Innovation Center for Cancer Medicine, Guangzhou 510275, China; ${ }^{2}$ Department \\ of Thoracic Surgery, Sun Yat-sen University Cancer Center, Guangzhou 510060, China; ${ }^{3}$ Department of Pathology, Shenzhen Maternity and Child \\ Healthcare Hospital, Shenzhen 518028, China; ${ }^{4}$ Department of Thoracic Surgery, The First Affiliated Hospital of Jinan University, Guangzhou \\ 510630, China; ${ }^{5}$ Department of Medical Oncology, Sun Yat-sen University Cancer Center, Guangzhou 510060, China \\ Contributions: (I) Conception and design: L Zhang, X Yu; (II) Administrative support: L Zhang, Y Lin; (III) Provision of study materials or patients: R \\ Zhang, T Yang, M Zhang; (IV) Collection and assembly of data: K Xi, Y Wen, G Wang; (V) Data analysis and interpretation: Z Wang, X Zhang; (VI) \\ Manuscript writing: All authors; (VII) Final approval of manuscript: All authors. \\ \#These authors contributed equally to this work. \\ Correspondence to: Dr. Lanjun Zhang. Department of Thoracic Surgery, Sun Yat-sen University Cancer Center, No. 651 Dongfeng Road East, \\ Guangzhou 510060, China. Email: zhanglj@sysucc.org.cn.
}

Background: Alpha-l-fucosidase (AFU) not only detects hepatocellular carcinoma (HCC) early but also is used as a clinical prognostic indicator of several malignant tumors. However, no study has investigated the prognostic significance of AFU in a cohort of patients with esophageal squamous cell carcinomas (ESCCs). Methods: A retrospective dataset that included 160 consecutive patients with early stage (pT1N0) ESCC who received surgery between January 2005 and December 2012 was analyzed to identify the prognostic value of serum AFU for overall survival (OS) by using Kaplan-Meier analysis and Cox multivariate regression modeling.

Results: The level of serum AFU ranged from 6.2 to $77.0 \mathrm{U} / \mathrm{L}$ with a median of $19.9 \mathrm{U} / \mathrm{L}$, and the best cutoff point for OS was 17.95 U/L. Analysis by Pearson's correlation showed that the levels of serum ALT and GGT were both positively correlated with the level of serum AFU ( $r=0.403, \mathrm{P}<0.001$ and $\mathrm{r}=0.264$, $\mathrm{P}=0.001$, respectively). After adjusting for significant factors identified by univariate analysis, the Cox multivariate regression model indicated that a young age ( $<65$ years), no history of alcohol consumption, and a low AFU level $(\leq 17.95 \mathrm{U} / \mathrm{L})$ were still significantly associated with longer OS $(\mathrm{P}=0.008,0.004$ and 0.017 , respectively). The 5-year and 10-year OS rates for patients with high AFU levels $v s$. low AFU levels were $76.2 \%$ vs. $86.0 \%$, and, $46.7 \%$ vs. $83.4 \%$, respectively.

Conclusions: Compared with other serum biomarkers, AFU showed a better prognostic value for longterm survival in patients with early stage ESCC.

Keywords: Esophageal squamous cell carcinoma (ESCC); alpha-l-fucosidase (AFU); prognosis

Submitted Apr 13, 2019. Accepted for publication Aug 06, 2019.

doi: $10.21037 /$ jtd.2019.08.92

View this article at: http://dx.doi.org/10.21037/jtd.2019.08.92

\section{Introduction}

Esophageal carcinoma (EC) is the ninth most common malignant tumor and causes the fifth highest number of cancer-related deaths worldwide (1). It is an endemic malignancy, particularly in the Asian esophageal cancer belt, and the incidence of EC varies in different countries and regions $(1,2)$. In China, the estimated number of new EC cases in 2015 was 477,900 , which accounted for more than half of new global cases (1). Despite tertiary prevention, 
guideline-concordant treatment, and standardized followup for EC established by the Chinese Society of Clinical Oncology (CSCO), the survival outcomes are still not ideal, and 5 -year overall survival (OS) rates ranged from $5 \%$ to $17 \%$ in recent reports $(1,3,4)$. Early detection and treatment provide a great opportunity to reduce death from cancers, such as colorectal cancer, gastric cancer, and non-small cell lung cancer $(3,5,6)$. However, the outcomes for some patients, even those with early stage EC (T1) treated with limited surgery, have not been favorable; in China, more than $15 \%$ of these patients died of cancer-related causes 5 years after diagnosis (compared with approximately $30 \%$ of those in the United States and $22.5 \%$ in Japan) $(2,4,5,7)$. Therefore, finding independent predictors to distinguish patients with poor survival is particularly important $(5,8)$. Individual multidisciplinary therapy (MDT) may be suitable for prolonging the survival of patients with early stage EC who also have high levels of other-risk factors $(2,5,7)$.

Increased or altered alpha-l-fucose-containing fucoglycoconjugates, such as glycolipids, glycoproteins, and mucoopolysaccharides etc., on cell surface are not only correlated with oncogenic transformation, lymphatic and vascular invasion, but also associated with the immune escape or tolerance. However, alpha-l-fucosidase (AFU), a liposomal enzyme, is widely present in mammalian cells and associated with key aspects of the degradation of alpha1-fucose. Consequently, the balance of alpha-1-fucose and AFU may affect the prognosis of malignancies. And theoretically, because AFU would be consumed during the degradation of alpha-1-fucose, patients with a low AFU level may have better survival (9-16). As a tumor biomarker, AFU was first introduced by Deugnier et al. in the diagnosis of hepatocellular carcinoma (HCC), and subsequent studies have also confirmed that serum AFU diagnosed HCC earlier than ultrasonography (9-11). Currently, the independent prognostic value of $\mathrm{AFU}$ for the postoperative survival of patients with HCC, colorectal carcinoma, intrahepatic cholangiocarcinoma and breast cancer has also been found (12-14). HCC patients with an elevated AFU level are more likely to die from cancer, but, possibly due to neoplastic heterogeneity, an elevated AFU level predicts a better OS rate among those with triple-negative breast cancer $(12,15)$.

However, to our knowledge, no available evidence has shown the feasibility of AFU as a prognostic factor in esophageal squamous cell carcinoma (ESCC). Thus, the aim of our study was first to explore the association between AFU and long-term survival in Chinese patients with early stage ESCC (pathological stage T1N0).

\section{Methods}

\section{Patient selection}

We retrospectively reviewed all patients who received surgery for pathological stage T1N0M0 (according to the 8th edition of the American Joint Committee on Cancer staging manual) ESCC at Sun Yat-sen University Cancer Center from January 2005 to December 2012. Then, patients were excluded from the preliminary database according to the following criteria: (I) received preoperative chemotherapy or chemoradiotherapy; (II) was diagnosed with secondary primary malignancy before or after the diagnosis of EC; (III) had a diagnosis of EC not otherwise specified (NOS); (IV) was diagnosed with other histology subtype(s), such as adenocarcinoma, small cell carcinoma or carcinosarcoma; (V) had no preoperative serum AFU examination; (VI) died within 30 days of their operation; (VII) were lost to follow-up. Finally, a total of 160 consecutive patients were retained in our study.

\section{Serum AFU measurement}

Serum AFU levels were measured within 1 week before the operation as previously described $(9,11,12)$. Fasting venous blood samples were taken from the patients and serum was obtained by centrifugation. A Hitachi Clinical Analyzer 7600 (Tokyo, Japan) was used to assess the AFU content in the serum within 24 hours after each blood sample was collected. During the interpretation, the normal reference range for serum AFU levels in our center was 5-40 U/L.

\section{Statistical analysis}

First, X-Tile software version 3.6.1 (Copyright Yale University 2003) was used to determine the best cutoff values for tumor length, resected lymph node count, alanine aminotransferase (ALT) level, aspartate aminotransferase (AST) level, gamma-glutamyl transferase (GGT) level, ALT/AST ratio, lactate dehydrogenase (LDH) level, and AFU level by the minimal $P$ value approach. Second, all statistical analyses in our study were performed using the Statistical Product and Service Solutions software package version 23.0 (SPSS Inc., Chicago, IL, USA). Among these, the Pearson Chi-square test was applied to the categorical variables to compare baseline characteristics 
between the low AFU group and the high AFU group, and an independent $\mathrm{t}$ test was used to compare continuous variables between the two groups. In addition, correlations from Pearson's linear correlation analysis are shown as scatter plots.

OS time was calculated from the day of ESCC diagnosis to death or the last follow-up (1 June 2018), but only patient occurred death was recorded as uncensored. In addition, the Kaplan-Meier method was used to plot the OS curve and for univariate analysis; all the statistically significant variables identified by univariate analysis were subsequently included in the COX multivariate regression model to explore potential prognostic indicators.

In addition, to ensure the consistency of the data and facilitate subsequent data processing and analysis, the alcohol use status was converted into digital data in our study based on the World Health Organization (WHO) Risk Drinking Levels, as shown in Table S1. Individuals with medium risk drinking or above were defined as alcohol consumption.

Following the well-known statistical principle, a $\mathrm{P}$ value less than 0.05 indicated statistical significance.

\section{Ethics statement}

The Research Data Deposit (RDD) Management Committee at Sun Yat-sen University Cancer Center has approved of our deposited research data, and the RDD number for this dataset is RDDA2018000855. In addition, our research was approved by the Institute Research Medical Ethics Committee at Sun Yat-sen University Cancer Center (approval number: B2014-110), and informed consent forms were signed by all patients.

\section{Results}

\section{The baseline characteristics and relationships between the two AFU groups}

From January 2005 to December 2012, a total of 160 consecutive patients with a pathologically confirmed diagnosis of pathological stage T1N0 ESCC were included in the final analysis. Their clinicopathological characteristics and hematological indexes are shown in Table 1. In our cohort, male patients comprised a large majority $(\mathrm{n}=105$, $65.6 \%$ ), and the median age at diagnosis was 59 years old (range, 32-82 years old). Interestingly, more than half of the patients $(\mathrm{n}=86,53.8 \%)$ reported tobacco exposure, but only
22 patients (13.8\%) had a history of alcohol consumption. In addition, primary carcinomas were located in the middle and lower thoracic esophagus at a high frequency $(65.0 \%$ and $18.7 \%$, respectively), and the Sweet procedure (left thoracic incision) was often chosen for the excision of esophageal neoplasms ( $\mathrm{n}=102,63.8 \%)$.

The levels of AFU ranged from 6.2 to $77.0 \mathrm{U} / \mathrm{L}$ with a median of $19.9 \mathrm{U} / \mathrm{L}$. The best cutoff point for OS was found to be $17.95 \mathrm{U} / \mathrm{L}$ by X-Tile software, and the area under the curve (AUC) was 0.591 in the receiver operating characteristic (ROC) curve (Figure 1A). The baseline characteristics in the patients with low AFU levels $(\leq 17.95 \mathrm{U} / \mathrm{L})$ and high AFU levels $(>17.95 \mathrm{U} / \mathrm{L})$ were compared (Table 2). A high AFU level (>17.95 U/L) was associated with a history of smoking $(\mathrm{P}=0.019)$, a high level of $\operatorname{ALT}(\mathrm{P}=0.012)$, and a high level of GGT $(\mathrm{P}=0.005)$. Additionally, the levels of ALT (Figure 1B) and GGT (Figure 1C) were positively associated with the level of AFU using Pearson's linear correlation analysis ( $r=0.403$ and 0.264 , respectively). However, there was no statistically significant difference in the distribution of other clinicopathological and hematological indexes between patients with high or low AFU levels (all $\mathrm{P}>0.05$, Table 2).

\section{Survival outcomes and prognostic analysis}

In our cohort, 42 patients $(42 / 160,26.3 \%)$ had occurred death at the last follow-up (1 June 2018), and 37 deaths of them were directly related with ESCC. Median follow-up time was 83.8 months (range, 64.0-107.5 months), and the 5 -year and 10-year OS rate for all 106 consecutive patients with pathological stage T1N0 ESCC was $79.4 \%$ and $66.3 \%$, respectively.

In univariate analysis, patients with a low AFU level $(\leq 17.95 \mathrm{U} / \mathrm{L})$ showed a significantly longer 10 -year OS than patients with a high AFU level (>17.95 U/L) $(83.4 \%$ and $46.7 \%, \mathrm{P}=0.028$, Table 1 and Figure 2). Additionally, univariate analysis also indicated that age, alcohol consumption, AST level, and LDH level were prognostic factors (Table 1). After Cox multivariable regression analysis, young patients ( $<65$ years), patients with no history of alcohol consumption, and patients with low AFU level $(\leq 17.95 \mathrm{U} / \mathrm{L})$ were still significantly associated with a longer OS (Table 1 and Figure 2).

\section{Discussion}

This study is the first to demonstrate and report that AFU 
Table 1 Baseline characteristics of all 160 patients with pathological stage T1N0 ESCC and analysis of the prognostic factors for OS

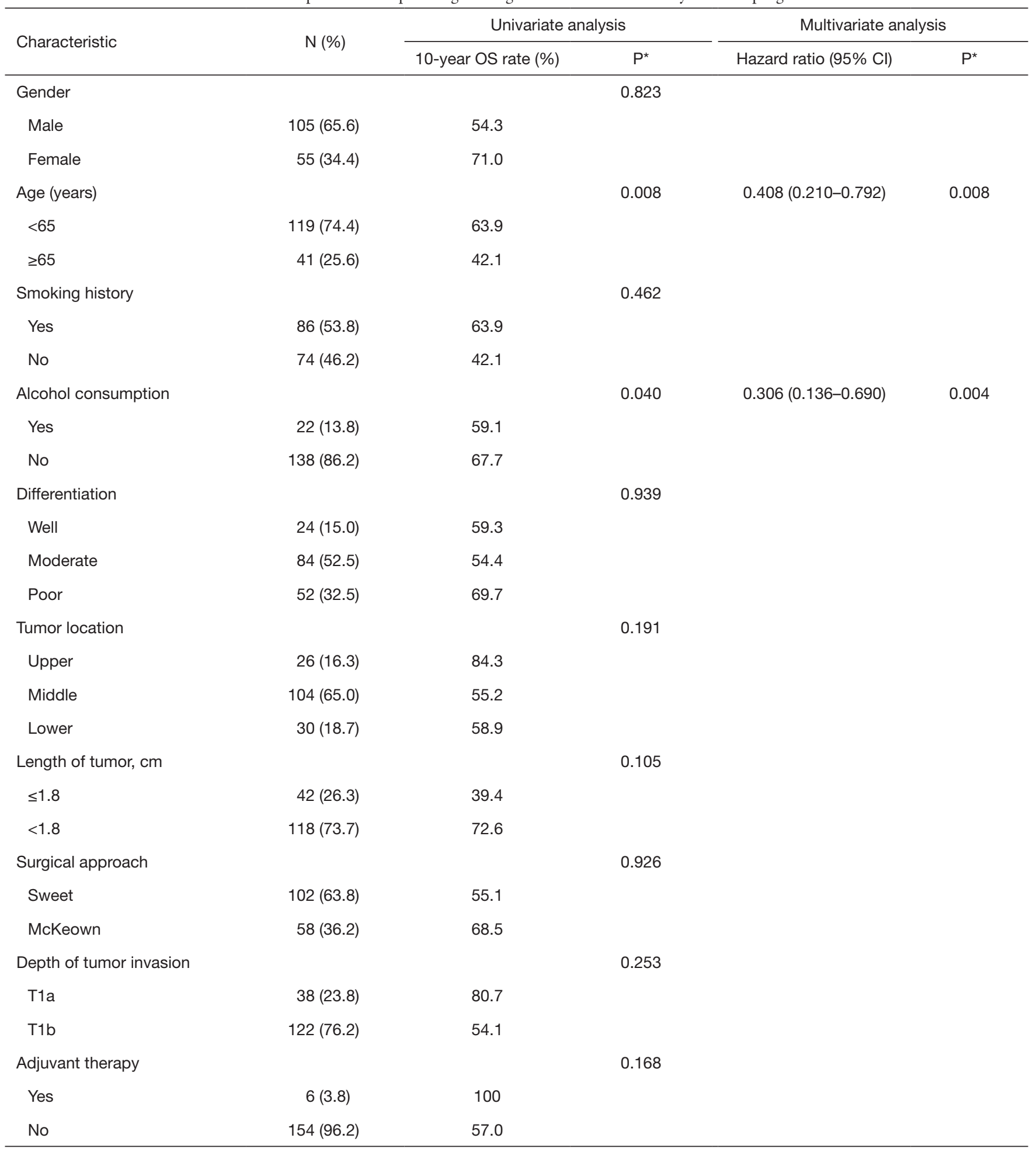

Table 1 (continued) 
Table 1 (continued)

\begin{tabular}{|c|c|c|c|c|c|}
\hline Characteristic & $\mathrm{N}(\%)$ & \multicolumn{2}{|c|}{ Univariate analysis } & \multicolumn{2}{|c|}{ Multivariate analysis } \\
\hline Resected lymph nodes count & & & 0.061 & $1.192(0.625-2.272)$ & 0.593 \\
\hline$\leq 13$ & $53(33.1)$ & 44.7 & & & \\
\hline$>13$ & $107(66.9)$ & 74.1 & & & \\
\hline$\leq 18.55$ & $88(55.0)$ & 59.0 & & & \\
\hline$>18.55$ & $72(45.0)$ & 57.6 & & & \\
\hline AST & & & $<0.001$ & $1.861(0.897-3.860)$ & 0.095 \\
\hline$\leq 15.35$ & 27 (16.9) & 34.9 & & & \\
\hline$\leq 0.75$ & $49(30.6)$ & 61.4 & & & \\
\hline$>0.75$ & $111(69.4)$ & 57.3 & & & \\
\hline GGT & & & 0.250 & & \\
\hline$\leq 21.85$ & $82(51.3)$ & 54.8 & & & \\
\hline$>21.85$ & $78(48.7)$ & 61.0 & & & \\
\hline LDH & & & 0.035 & $2.215(0.924-5.308)$ & 0.075 \\
\hline$\leq 170.20$ & $108(67.5)$ & 53.6 & & & \\
\hline$>170.20$ & $52(32.5)$ & 58.7 & & & \\
\hline
\end{tabular}

* $\mathrm{P}$ value less than 0.05 indicated a statistically significant difference. ESCC, esophageal squamous cell carcinoma; OS, overall survival; ALT, alanine aminotransferase; AST, aspartate aminotransferase; GGT, gamma-glutamyl transferase; LDH, lactate dehydrogenase; AFU, alpha-I-fucosidase.

significantly and independently predicts prognosis in patients with ESCC. Patients with pathological stage T1N0 ESCC who had low AFU levels ( $\leq 17.95 \mathrm{U} / \mathrm{L}$ ) had a significantly longer 10-year OS than patients with high AFU levels (>17.95 U/L) (83.4\% and 46.7\%, P=0.028). Furthermore, a high AFU level was associated with a history of smoking, a high level of ALT, and a high level of GGT.

As a tumor biomarker, AFU was first introduced by Deugnier et al. in the diagnosis of HCC, and subsequent studies have also confirmed that serum AFU diagnosed HCC earlier than ultrasonography (9-11). Fawzy Montaser and colleagues found that AFU examination detected abnormalities earlier than abnormal ultrasonography imaging observable at 6-9 months (12). In a meta-analysis from Gan et al., the pooled sensitivity of AFU for the diagnosis of HCC was 0.72 while the pooled specificity was 0.78 , and the AUC was 0.8125 . They concluded that AFU could be used as a tumor marker for the diagnosis of HCC with great value in clinical application (16). Currently, the independent prognostic value of AFU levels for postoperative survival of patients with HCC, colorectal carcinoma and breast cancer has also been reported (11-16). HCC and colorectal carcinoma patients with an elevated AFU level are more likely to die from cancer, but an elevated AFU level predicts a better OS rate among triplenegative breast cancer patients. In our research, similar to 
A

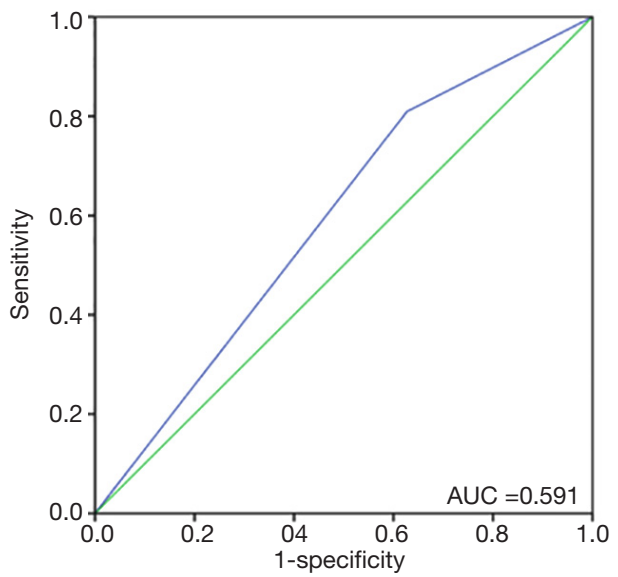

C

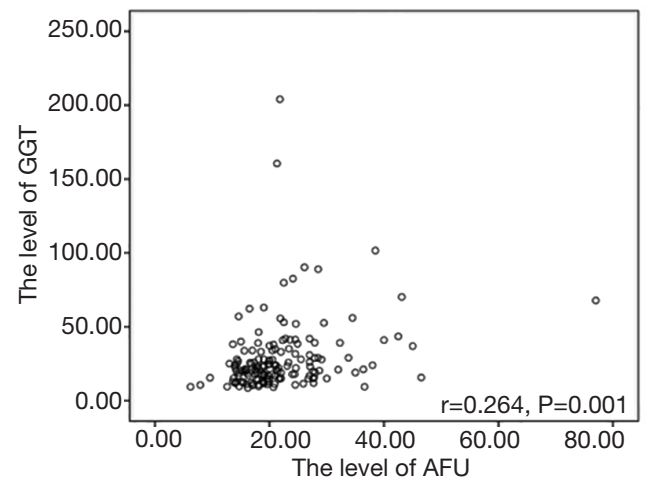

B

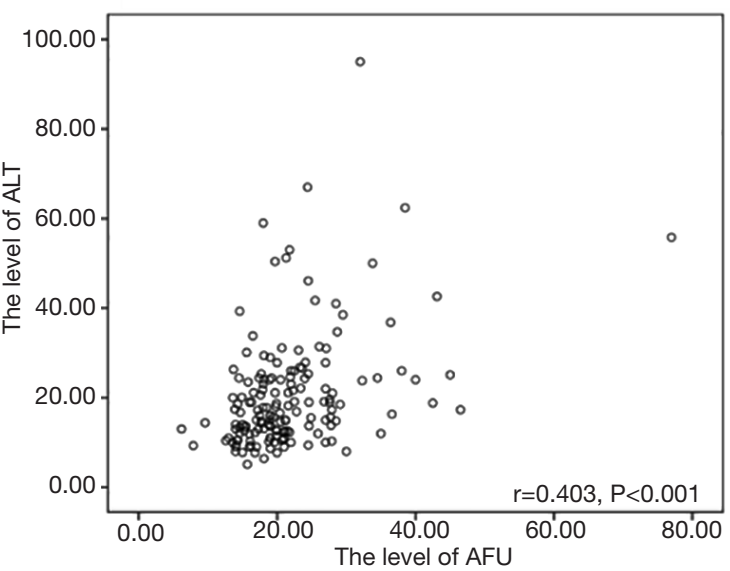

Figure 1 The predictive value of AFU in long-term survival and Pearson's linear correlation analysis. (A) The receiver operating characteristic curve of the AFU; (B) distribution of the levels of AFU and ALT; (C) distribution of the levels of AFU and GGT. AFU, alpha1-fucosidase; ALT, alanine aminotransferase; GGT, gamma-glutamyl transferase.

the results in patients with HCC and colorectal carcinoma, the AFU levels were similarly able to predict the prognosis of pathological stage T1N0 ESCC patients (11,12,14-16).

A review on the mechanism of AFU revealed that an increased fucose or fucosylation content and aberrant fucosylation on the membrane surface of tumor cells may be associated with the metastatic capacity of tumors and could help abnormal cells escape immunological recognition (17-20). Thus, fucose expression or fucosylation might play a critical role in tumor progression and poor survival (17-19). However, these fucosylated glycoconjugates could be degraded by $\mathrm{AFU}$, which would maintain a lower fucose content, prevent tumor metastasis, and induce immune responses (17-20). Accordingly, the serum AFU level, which would be consequently consumed, decreasing the fucosylated glycoconjugates synthesized by the neoplasm, could be used to predict the preoperative survival of patients with malignant tumors $(17,19)$. Although previous studies have reported the level of AFU activity in malignant tumors to be positively correlated with TNM staging or Duke's stage, low AFU serum content in early stage patients still precisely reflects tumor load and the degree of malignancy $(14,15)$. In addition, AFU may be a potential therapeutic molecular target for clinical applications $(13,17,18,20)$.

As specific, inexpensive and easily available biomarkers, ALT, AST, GGT and LDH are generally used in the preoperative assessment of liver function (21). In recent years, increasing studies have noted that these biomarkers are not only related to the risk of malignant tumor occurrence, but also associated with survival after antitumor treatment for cancers, such as gastric cancer, breast cancer, liver cancer, ESCC and other cancers (22-24). In a retrospective study, Huang et al. reported that ESCC cases with a lower ALT/AST ratio showed a worse prognosis than cases with a higher ratio $(\mathrm{P}<0.001)$; this was observed not only in the entire cohort but also in both the T1-2 and N0 subgroups (22). However, the predictive value of the ALT/AST ratio for long-term survival was not found in our 
Table 2 The relationships between AFU levels and baseline characteristics in patients with stage T1N0 ESCC

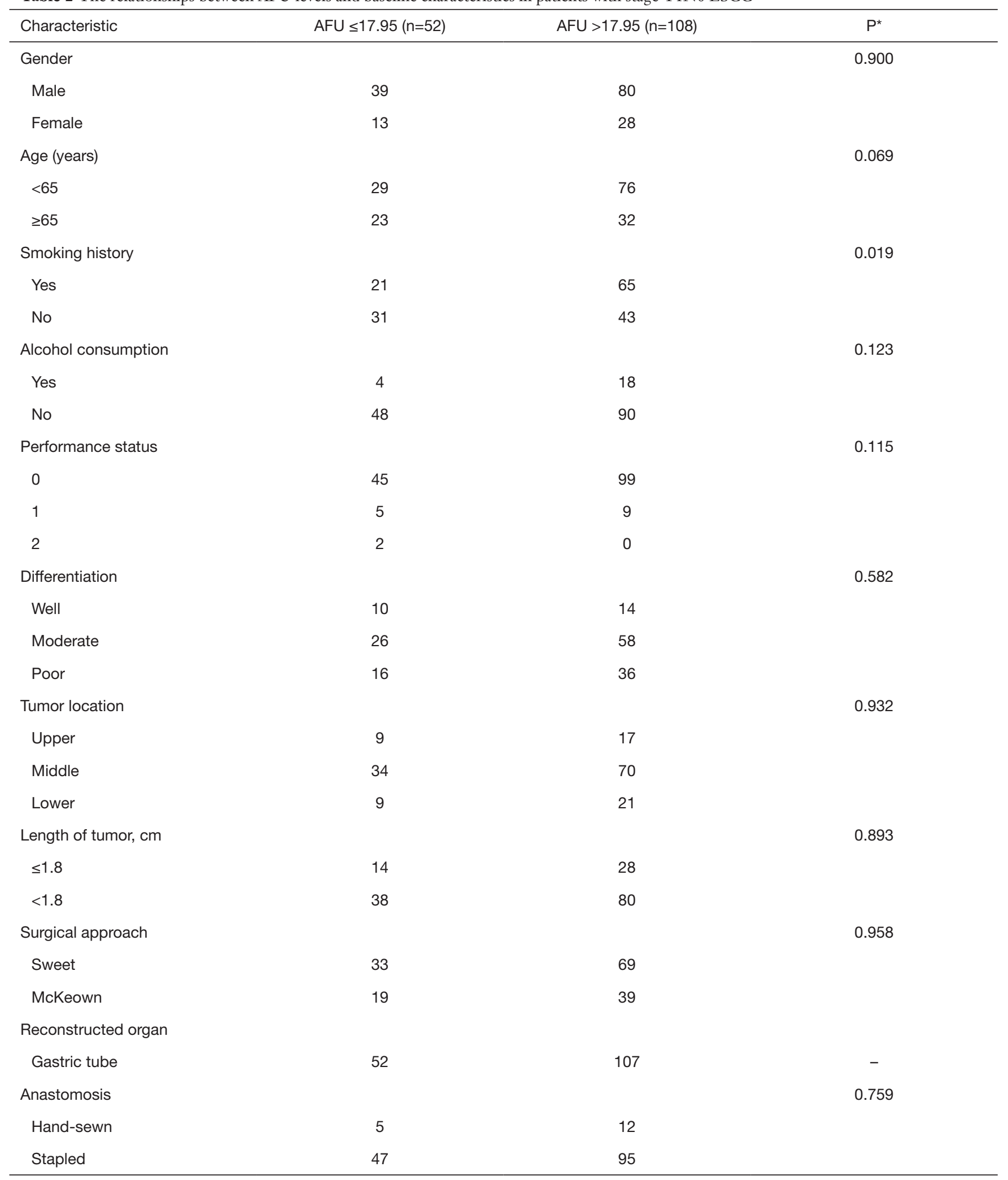

Table 2 (continued) 
Table 2 (continued)

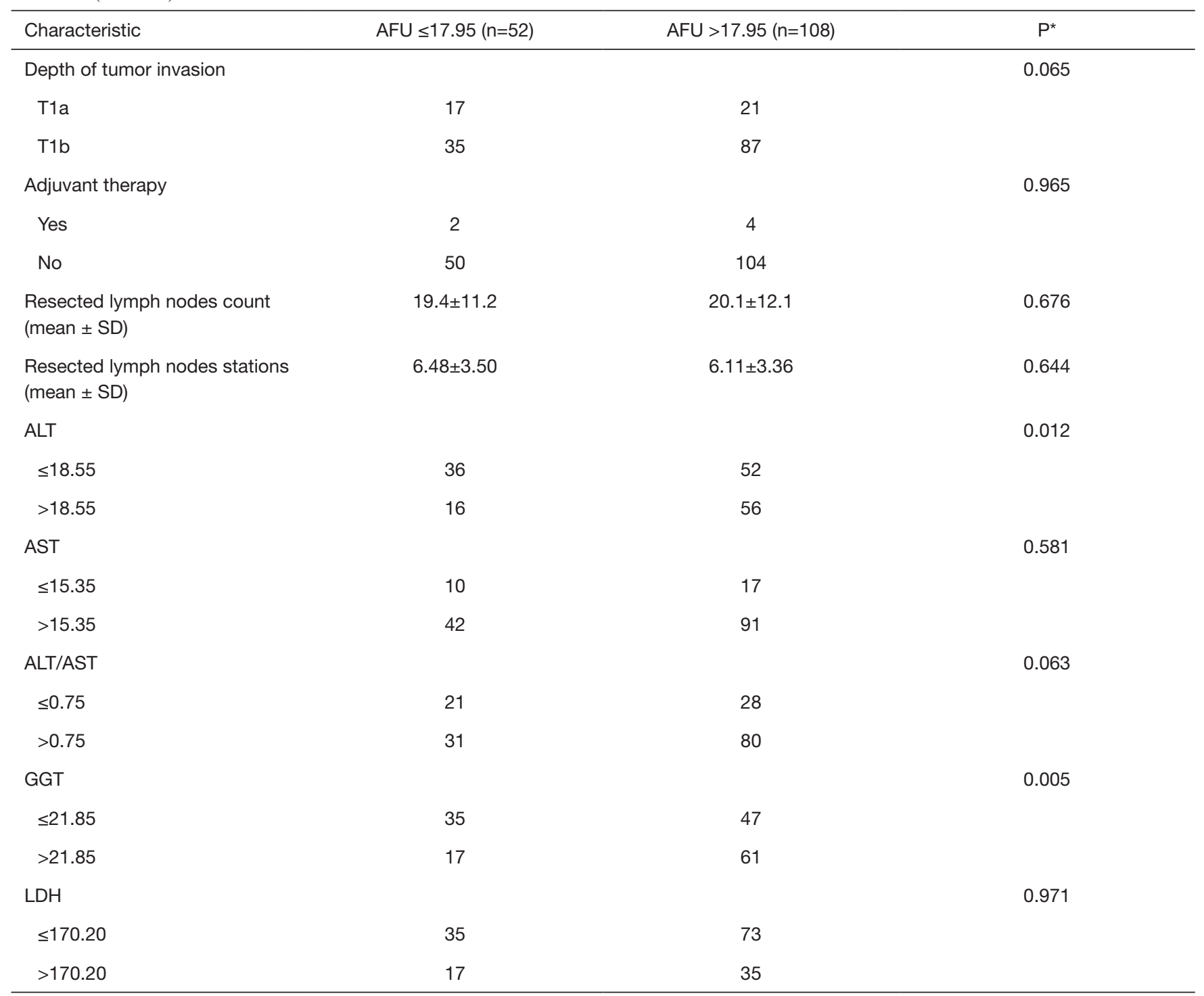

*, P value less than 0.05 indicated a statistically significant difference. ESCC, esophageal squamous cell carcinoma; OS, overall survival; ALT, alanine aminotransferase; AST, aspartate aminotransferase; GGT, gamma-glutamyl transferase; LDH, lactate dehydrogenase; AFU, alpha-l-fucosidase.

patients with pathological stage T1N0 ESCC. In addition, unlike results in hepatitis B-induced HCC, the level of AST or ALT alone was not related to the clinical outcome of ESCC, and this was shown in not only a previous report but also our study $(22,23)$. Recently, the prognostic capabilities of GGT and LDH in ESCC have also been widely demonstrated, and the cases enrolled in these related studies included all TNM stages (I-IV) (22-25). However, in adjusted survival analysis, these two biomarkers did not play significant prognostic roles in our study, which was consistent with a subgroup analysis from Huang et al. (22). Possible reasons for the differences described above include the following.

First, when used to assess liver function, these biomarkers could also indicate anti-inflammatory and anti-oxidant properties, exposure to certain carcinogens, and alcohol intake $(26,27)$. Accordingly, higher levels of these biomarkers were positively associated with more severe systemic inflammatory response syndrome (SRS), a more advanced stage and distant metastasis, as indicated 
A

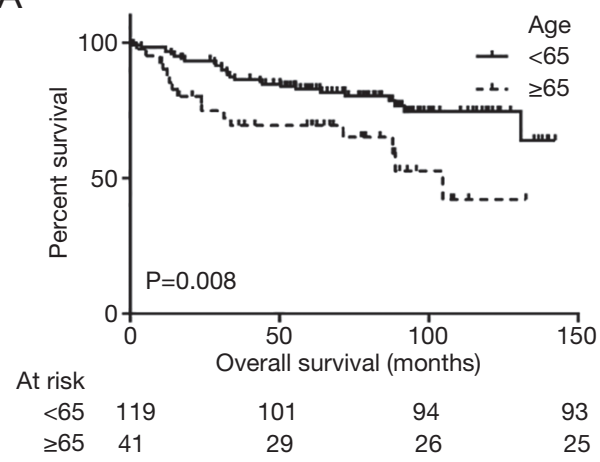

B

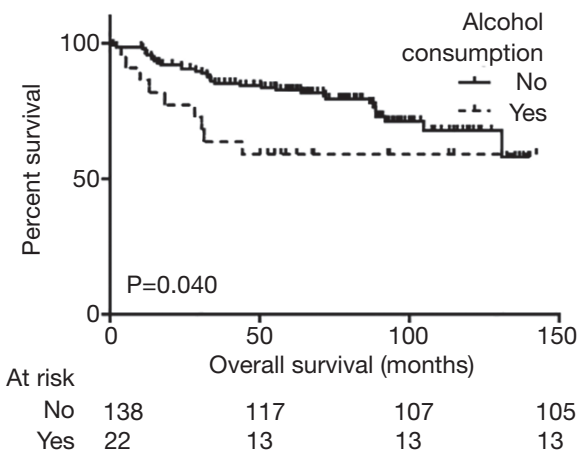

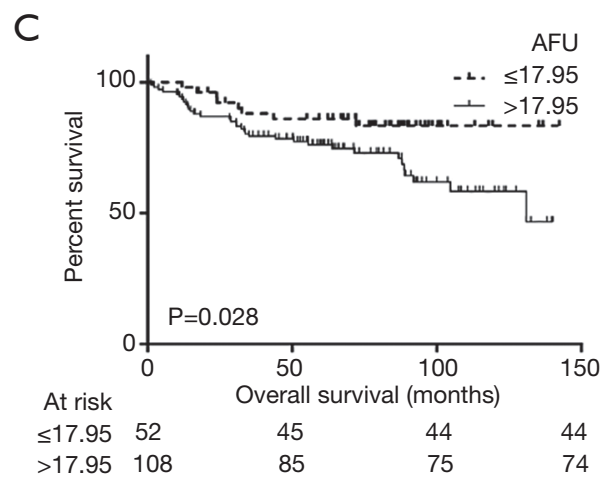

Figure 2 Kaplan-Meier survival curves for patients with pathological stage T1N0 ESCC according to (A) age at diagnosis, (B) alcohol consumption, (C) the level of serum AFU. ESCC, esophageal squamous cell carcinoma.

by numerous studies (21-25). In other words, few of these enzymes were released by active inflammatory cells or tumor cells in early stage carcinoma; hence, their levels were not sufficient to distinguish prognostic differences in the population with a lower tumor load $(15,22)$. Second, alcohol intake was positively related to the serum content of these liver function indexes, especially the ALT/AST ratio and GGT, which was also validated in our study ( $\mathrm{P}=0.048$ and 0.031 , respectively; compared by independent $t$ test $)(22,25)$. However, current or former alcohol consumption was an uncommon phenomenon in our cohort (22/160, 13.8\%); therefore, the serum contents of these liver function indexes remained low, and no significant individual differences were found (26). Third, our research was carried out at a single center with a small number of patients from a retrospective database, this likely leading to selection bias, which was also one of the limitations of this study.

To date, little data was available regarding the correlation between serum AFU level and liver dysfunction. Reported by Cheng et al. in a large-scale study in HCC following hepatectomy, the positive correlation between the level of AFU and AFP was found (9). Recently, in a similar prognosis study, $\mathrm{Li}$ and his coworkers tried to explore the correlation between the level of AFU and transaminase (including ALT and AST) in intrahepatic cholangiocarcinoma, but no significant correlation was detected. In this study, we first found that a high AFU level was associated with a high level of ALT and GGT in ESCC, and the possible explanations for the relationships remained unclear. However, after multivariable regression analysis, only the AFU was proved to be independent prognostic index with OS. This was exactly the argument that supports our conclusion: serum AFU showed a better prognostic value than other liver function index for long-team survival in patients with early stage ESCC.

This is the first study to explore the association between AFU levels and long-term survival in patients with ESCC, and the prognostic value of serum AFU content in early stage malignant tumors was also demonstrated and reported (9-15). However, several limitations of this study should be noted when interpreting the results. First, the study spanned a long period of time (almost 10 years), 
and much progress in testing, surgical procedures, and perioperative management occurred during that time $(9,11,17,19,20)$. Second, the results might be unreliable given current attitudes. In addition, chronic hepatitis viral infection and alcoholic hepatitis are common phenomena in China, likely leading to abnormal non-neoplasm-related liver function $(27,28)$. Another obvious limitation of this study was our selection of the best cutoff value, because a different definition of high AFU content might lead to a different result, which might weaken the clinical application of this novel biomarker (9-15) . Even so, the potential prognostic value of serum AFU content in ESCC has been demonstrated, and future research directions regarding molecular targets for ESCC treatment have also been proposed.

\section{Conclusions}

In conclusion, compared with other serum biomarkers, serum AFU levels showed a better prognostic value for long-team survival in patients with early stage ESCC. This enzyme may be a potential therapeutic molecular target for ESCC.

\section{Acknowledgments}

Funding: This study was funded by the National Key Research and Development Plan (No. 2016YFC0905400), Ministry of Science and Technology of the People's Republic of China.

\section{Footnote}

Conflicts of Interest: The authors have no conflicts of interest to declare.

Ethical Statement: The authors are accountable for all aspects of the work in ensuring that questions related to the accuracy or integrity of any part of the work are appropriately investigated and resolved. This retrospective study was approved by the board-certified Research Ethics Committee at our center (No. B2014-110) and written informed consent was obtained from all patients.

\section{References}

1. Chen $\mathrm{W}$, Zheng R, Baade PD, et al. Cancer statistics in China, 2015. CA Cancer J Clin 2016;66:115-32.
2. Kitagawa Y, Uno T, Oyama T, et al. Esophageal cancer practice guidelines 2017 edited by the Japan Esophageal Society: part 1. Esophagus 2019;16:1-24.

3. Wu L, Wang C, Tan X, et al. Radiomics approach for preoperative identification of stages I-II and III-IV of esophageal cancer. Chin J Cancer Res 2018;30:396-405.

4. Tang WR, Fang JY, Wu KS, et al. Epidemiological characteristics and prediction of esophageal cancer mortality in China from 1991 to 2012. Asian Pac J Cancer Prev 2014;15:6929-34.

5. Gao QY, Fang JY. Early esophageal cancer screening in China. Best Pract Res Clin Gastroenterol 2015;29:885-93.

6. Kumagai Y, Tachikawa T, Higashi M, et al. Vascular endothelial growth factors $\mathrm{C}$ and $\mathrm{D}$ and lymphangiogenesis at the early stage of esophageal squamous cell carcinoma progression. Dis Esophagus 2018. doi: 10.1093/dote/ doy011.

7. Enzinger PC, Mayer RJ. Esophageal cancer. N Engl J Med 2003;349:2241-52.

8. Teramoto H, Koike M, Tanaka C, et al. Tumor budding as a useful prognostic marker in T1-stage squamous cell carcinoma of the esophagus. J Surg Oncol 2013;108:42-6.

9. Deugnier Y, David V, Brissot P, et al. Serum alpha-Lfucosidase: a new marker for the diagnosis of primary hepatic carcinoma? Hepatology 1984;4:889-92.

10. Leray G, Deugnier Y, Jouanolle AM, et al. Biochemical aspects of alpha-L-fucosidase in hepatocellular carcinoma. Hepatology 1989;9:249-52.

11. Wang K, Guo W, Li N, et al. Alpha-1-fucosidase as a prognostic indicator for hepatocellular carcinoma following hepatectomy: a large-scale, long-term study. Br J Cancer 2014;110:1811-9.

12. Fawzy Montaser M, Amin Sakr M, Omar Khalifa M. Alpha-L-fucosidase as a tumour marker of hepatocellular carcinoma. Arab J Gastroenterol 2012;13:9-13.

13. Cheng TC, Tu SH, Chen LC, et al. Down-regulation of alpha-L-fucosidase 1 expression confers inferior survival for triple-negative breast cancer patients by modulating the glycosylation status of the tumor cell surface. Oncotarget 2015;6:21283-300.

14. Ayude D, Paez De La Cadena M, Martinez-Zorzano VS, et al. Preoperative serum alpha-L-fucosidase activity as a prognostic marker in colorectal cancer. Oncology 2003;64:36-45.

15. Delacadena M, Fernandez J, Decarlos A, et al. Low levels of alpha-L-fucosidase activity in colorectal cancer are due to decreased amounts of the enzymatic protein and are related with Dukes' stage. Int J Oncol 1996;9:747-54. 
16. Gan Y, Liang Q, Song X. Diagnostic value of alpha-Lfucosidase for hepatocellular carcinoma: a meta-analysis. Tumour Biol 2014;35:3953-60.

17. Muramatsu T. Carbohydrate signals in metastasis and prognosis of human carcinomas. Glycobiology 1993;3:291-6.

18. Thompson S, Dargan E, Turner GA. Increased fucosylation and other carbohydrate changes in haptoglobin in ovarian cancer. Cancer Lett 1992;66:43-8.

19. Wright LC, May GL, Gregory P, et al. Inhibition of metastatic potential by fucosidase: an NMR study identifies a cell surface metastasis marker. J Cell Biochem 1988;37:49-59.

20. Byrne AM, Sharma R, Duggan G, et al. Deoxycholic acid impairs glycosylation and fucosylation processes in esophageal epithelial cells. Glycobiology 2012;22:638-48.

21. Brockmoller J, Roots I. Assessment of liver metabolic function. Clinical implications. Clin Pharmacokinet 1994;27:216-48.

22. Huang H, Wang XP, Li XH, et al. Prognostic value of pretreatment serum alanine aminotransferase/ aspartate aminotransferase (ALT/AST) ratio and gamma glutamyltransferase (GGT) in patients with esophageal

Cite this article as: Yu X, Zhang R, Yang T, Zhang M, Xi K, Lin Y, Wen Y, Wang G, Huang Z, Zhang X, Zhang L. Alphal-fucosidase: a novel serum biomarker to predict prognosis in early stage esophageal squamous cell carcinoma. J Thorac Dis 2019;11(9):3980-3990. doi: 10.21037/jtd.2019.08.92 squamous cell carcinoma. BMC Cancer 2017;17:544.

23. Wei XL, Zhang DS, He MM, et al. The predictive value of alkaline phosphatase and lactate dehydrogenase for overall survival in patients with esophageal squamous cell carcinoma. Tumour Biol 2016;37:1879-87.

24. Shen SL, Fu SJ, Chen B, et al. Preoperative aspartate aminotransferase to platelet ratio is an independent prognostic factor for hepatitis B-induced hepatocellular carcinoma after hepatic resection. Ann Surg Oncol 2014;21:3802-9.

25. Whitfield JB. Gamma glutamyl transferase. Crit Rev Clin Lab Sci 2001;38:263-355.

26. Teschke R, Rauen J, Neuefeind M, et al. Alcoholic liver disease associated with increased gammaglutamyltransferase activities in serum and liver. Adv Exp Med Biol 1980;132:647-54.

27. Griswold MG, Fullman N, Hawley C. Alcohol use and burden for 195 countries and territories, 1990-2016: a systematic analysis for the Global Burden of Disease Study 2016. Lancet 2018;392:1015-35.

28. Yu R, Fan R, Hou J. Chronic hepatitis B virus infection: epidemiology, prevention, and treatment in China. Front Med 2014;8:135-44. 
Supplementary

Table S1 World Health Organization (WHO) level of drinking risk (in 2014)

\begin{tabular}{lcc}
\hline \multirow{2}{*}{ WHO risk level } & \multicolumn{2}{c}{ Grams (g) of pure alcohol per day } \\
\cline { 2 - 3 } & Female & 0 \\
\hline Abstinence & 0 & $1-40$ \\
Low risk & $1-20$ & $41-60$ \\
Medium risk & $21-40$ & $61-100$ \\
High risk & $41-60$ & $101+$ \\
Very high risk & $61+$ & $101+$ \\
\hline
\end{tabular}

